Paper prepared for $50^{\text {th }}$ AIAA Aerospace Sciences Meeting to be held on 9-12 January, 2012 in Nashville, Tennessee

\title{
Implementation of Finite Volume based Navier Stokes Algorithm within General Purpose Flow Network Code
}

\author{
Paul Schallhorn \\ NASA/Kennedy Space Center \\ Florida - 32899
\& Alok Majumdar
NASA/Marshall Space Flight Center
Huntsville, Alabama - 35812

\begin{abstract}
This paper describes a finite volume based numerical algorithm that allows multi-dimensional computation of fluid flow within a system level network flow analysis. There are several thermo-fluid engineering problems where higher fidelity solutions are needed that are not within the capacity of system level codes. The proposed algorithm will allow NASA's Generalized Fluid System Simulation Program (GFSSP) to perform multi-dimensional flow calculation within the framework of GFSSP's typical system level flow network consisting of fluid nodes and branches. The paper presents several classical two-dimensional fluid dynamics problems that have been solved by GFSSP's multi-dimensional flow solver. The numerical solutions are compared with the analytical and benchmark solution of Poiseulle, Couette and flow in a driven cavity.
\end{abstract}

\section{INTRODUCTION}

In thermo-fluid engineering applications, system level codes are typically used to find flow and pressure distribution in a complex flow network. On the other hand, Navier-Stokes codes are used when detailed knowledge about the flow is needed for design or investigate a failure scenario. System level codes are often run independently to provide boundary conditions for Navier Stokes codes. There has not been much success in the integration of these codes to perform any coupled analysis. However, there are situations where integrated analysis brings value to the design. One such example is the propellant feed to a rocket engine from a stratified cryogenic tank. While the bulk of the feed system analysis can be performed by a system level flow network code, the stratification is a multi-dimensional phenomenon and requires NavierStokes analysis in conjunction with network flow analysis which models pressurization and venting. 
In order to analyze such problems, it was decided to expand NASA's Generalized Fluid System Simulation Program (GFSSP) [1] to account for multidimensional flow effects. GFSSP is a finite volume based [2] network flow analysis code where a complex flow network is discretized into nodes and branches. The mass, energy and species conservation equations are solved at the nodes while momentum equations are solved at the branches. The one dimensional momentum equation in a flow network typically consists of pressure gradient, friction and fluid acceleration terms. In this paper the momentum equation has been extended to include shear due to velocity gradient and transport of longitudinal momentum due to transverse velocity component from neighboring branches in order to account for multi-dimensional effect of the flow. The unstructured nature of the code has been preserved so as to retain flexibility for irregular geometries.

\section{MATHEMATICAL FORMULATION \& VERIFICATION OF RESULTS}

GFSSP employs an upwind differencing scheme to derive the conservation equations of mass, momentum, energy and species conservation and solves the system of equations by a combination of successive substitution scheme and simultaneous (Newton-Raphson) scheme [3]. This section describes the extension of one-dimensional momentum equation to include a) the shear term in laminar flows due to velocity gradient, and b) transport of longitudinal momentum due to transverse velocity component from neighboring branches.

\section{Shear term in momentum equation}

The two-dimensional, steady-state Navier-Stokes Equations in rectangular Cartesian co-ordinate can be written as:

$\rho\left(u \frac{\partial u}{\partial x}+v \frac{\partial u}{\partial y}\right)=\frac{\partial(\rho u u)}{\partial x}+\frac{\partial(\rho v u)}{\partial y}=-\frac{\partial p}{\partial x}+\rho g_{x}+\mu\left(\frac{\partial^{2} u}{\partial x^{2}}+\frac{\partial^{2} u}{\partial y^{2}}\right)$

The first term on the left hand side of equation (1) corresponds to the longitudinal acceleration (inertia) term within GFSSP. The second term on the left hand side of equation (1) corresponds to the transverse momentum exchange. The first term on the right hand side of equation (1) corresponds to the pressure term within GFSSP. The second term on the right hand side of equation (1) corresponds to the gravity term within GFSSP. The third term on the right hand side of equation (1) is negligible (based on an order of magnitude argument). The fourth term on the right hand side of equation (1) corresponds to the friction term of GFSSP. It is this fourth term that represents the shear term that is sought.

The discretized form of shear term is expressed in equation 2. 


$$
\mu \frac{\partial^{2} \mathrm{u}}{\partial \mathrm{y}^{2}} \mathrm{~V} \approx \mu \frac{\Delta \mathrm{u}}{(\Delta \mathrm{y})^{2}}(\Delta \mathrm{x})(\Delta \mathrm{y})(\Delta \mathrm{z})=\mu \frac{\Delta \mathrm{u}}{\Delta \mathrm{y}} \Delta \mathrm{x} \Delta \mathrm{z}=\mu \frac{\Delta \mathrm{u}}{\Delta \mathrm{y}} \mathrm{A}_{\text {shear }}
$$

Figure 1, below, represents a set of nodes and branches for which shear forces are exchanged. Let branch 12 represent the branch for which the shear force is to be calculated. Branches N12 and S12 represent the parallel branches which will be used to calculate the shear force on branch 12. Let YS be the distance between branches 12 and S12, and let YN be the distance between branches 12 and N12. Let AS be the shearing area between branches 12 and S12, while AN is the shearing area between branches 12 and N12.

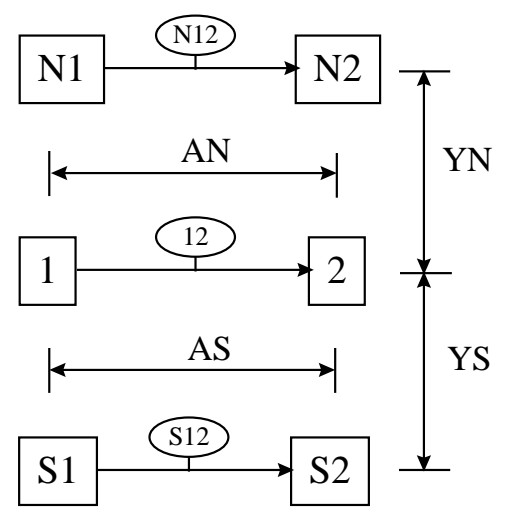

\section{Figure 1: Grid for Shear Exchange}

It is possible (and highly probable) that YS \& YN will not be the same nor exactly parallel to branch 12, therefore, a modified differencing scheme must be used. Equation (3) below is the proposed differencing scheme to be implemented into GFSSP:

$$
\mu \frac{\partial \mathrm{u}}{\partial \mathrm{y}} \mathrm{A}_{\text {Branch 12 } 2 \text { shear }} \approx\left[\left(\mu \frac{\mathrm{u}_{\mathrm{N} 12} \cos \theta_{\mathrm{N} 12}-\mathrm{u}_{12}}{\mathrm{YN}} \mathrm{AN}\right)+\left(\mu \frac{\mathrm{u}_{12}-\mathrm{u}_{\mathrm{S} 12} \cos \theta_{\mathrm{S} 12}}{\mathrm{YS}} \mathrm{AS}\right)\right]
$$

Equation (3) can be generalized to n-number of parallel branches at any position around branch 12 as shown in equation (4).

$\mu \frac{\partial \mathrm{u}}{\partial \mathrm{y}} \mathrm{A}_{\text {Branch 12 shear }} \approx \sum_{\mathrm{i}=1}^{\mathrm{n}}\left(\mu \frac{\mathrm{u}_{\mathrm{i} 12} \cos \theta_{\mathrm{i} 12}-\mathrm{u}_{12}}{\mathrm{Yi}} \mathrm{Ai}\right)$

where the summation subscript i represents the $i^{\text {th }}$ parallel branch to branch 12 .

The expression for the shear effect of the wall on branch 12 is given in equation (5). 




If there were multiple walls adjacent to branch 12 , then equation (5) could be generalized into equation (6).

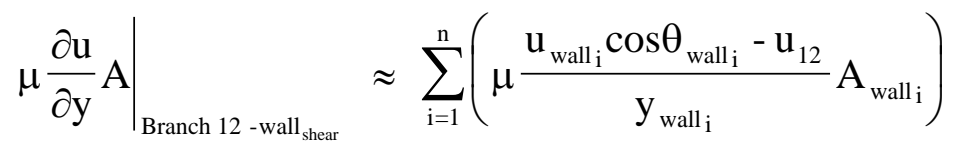

Equation (7) represents the laminar shear formulation where $\mathrm{i}$ is the current branch, $\mathrm{np}_{\mathrm{i}}$ is the number of parallel branches to branch $\mathrm{i}$, and $\mathrm{ns}_{\mathrm{i}}$ is the number of parallel solid walls to branch $\mathrm{i}$.

$$
\mu \frac{\partial \mathrm{u}}{\partial \mathrm{y}} \mathrm{A}_{\text {Branch i shear }} \approx \sum_{\mathrm{j}=1}^{\mathrm{np}}\left(\mu \frac{\mathrm{u}_{\mathrm{ij}} \cos \theta_{\mathrm{ij}}-\mathrm{u}_{\mathrm{i}}}{\mathrm{Y}_{\mathrm{ij}}} \mathrm{A}_{\mathrm{ij}}\right)+\sum_{\mathrm{k}=1}^{\mathrm{ns}_{\mathrm{i}}}\left(\mu \frac{\mathrm{u}_{\text {wall }_{\mathrm{ik}}} \cos \theta_{\text {wall }_{\mathrm{ik}}}-\mathrm{u}_{\mathrm{i}}}{\mathrm{y}_{\text {wall }_{\mathrm{ik}}}} \mathrm{A}_{\mathrm{ik}}\right)
$$

Let's examine two situations which will benchmark the laminar shear term of equation (7). The first situation is that of Poiseulle flow between infinite flat plates. The second situation is that of Couette flow between infinite parallel flat plates. The implementation of equation 7 in GFSSP has been verified by comparing its solution with two bench mark laminar flow examples: Poiseulle Flow and Couette Flow

\section{POISEULLE FLOW}

Consider the flow between two fixed flat plates shown below in Figure 2. The flow is pressure driven and assumed to be fully developed. The length of the plates is 1000 inches, they are 1 inch apart, the fluid density is $12 \mathrm{lb}_{\mathrm{m}} / \mathrm{ft}^{3}$, the viscosity is $1 \mathrm{lb} /(\mathrm{ft} \cdot \mathrm{sec})$, and the pressures at the upstream and downstream ends are 20 psi and 10 psi, respectively. Figure 3 shows an approximate velocity profile for this situation.



Figure 2: Poiseulle Flow Physical Situation 


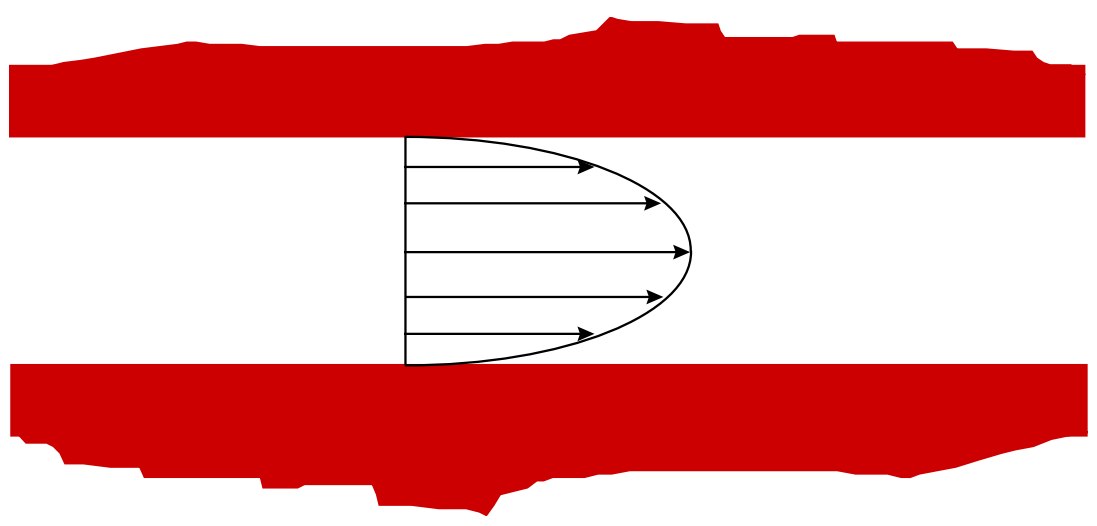

Figure 3: Poiseulle Flow Velocity Profile

The analytical solution of velocity for Poiseulle flow [4] for situation shown in figure 2 can be expressed as:

$$
\underline{u}=0.005\left(\mathrm{y}-\mathrm{y}^{2}\right) .
$$

\section{GFSSP model of Poiseulle Flow}

A simple 3 node, 10 branch model (two sets of 5 parallel branches) was constructed to model the physical situation described above. The model is shown schematically in Figure 4 below.

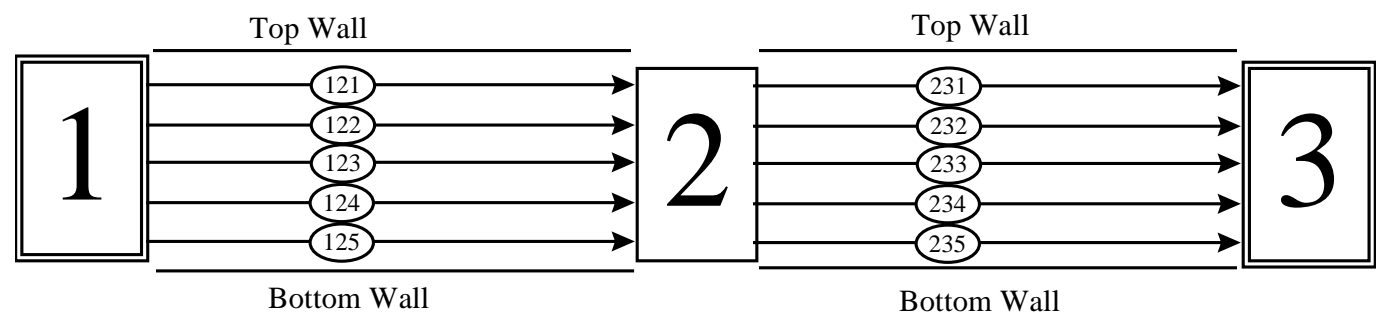

\section{Figure 4: GFSSP Model of Poiseulle Flow}

Each of the 10 branches $(121-125,231-235)$ are 500 inches in length. It is assumed that the shear area for each branch-branch \& branch-wall was $500 \mathrm{in}^{2}$ and that the shear distance was 0.2 inches between adjacent branches and 0.1 inch between walls and their adjacent branches. The pressures at nodes 1 and 3 are 20 psi and 10 psi, respectively. A constant density option was used and the corresponding density and viscosity used were $12 \mathrm{lb}_{\mathrm{m}} / \mathrm{ft}^{3}$ and $1.0 \mathrm{lb} /\left(\mathrm{ft}^{*} \mathrm{sec}\right)$, respectively. Resistance option -02 was used in the initial flow field calculation (for the first 
Newton-Raphson iteration, after which the shear will replace the friction factor calculation) for each of the branches. The bottom and top walls are fixed.

Figure 5 below shows a comparison between the velocity profiles for the analytical solution and the GFSSP 3 node, 10 branch (5 parallel branch) model. As can be seen in Figure 5, the results of this crude GFSSP model compare very favorably with the analytical solution.

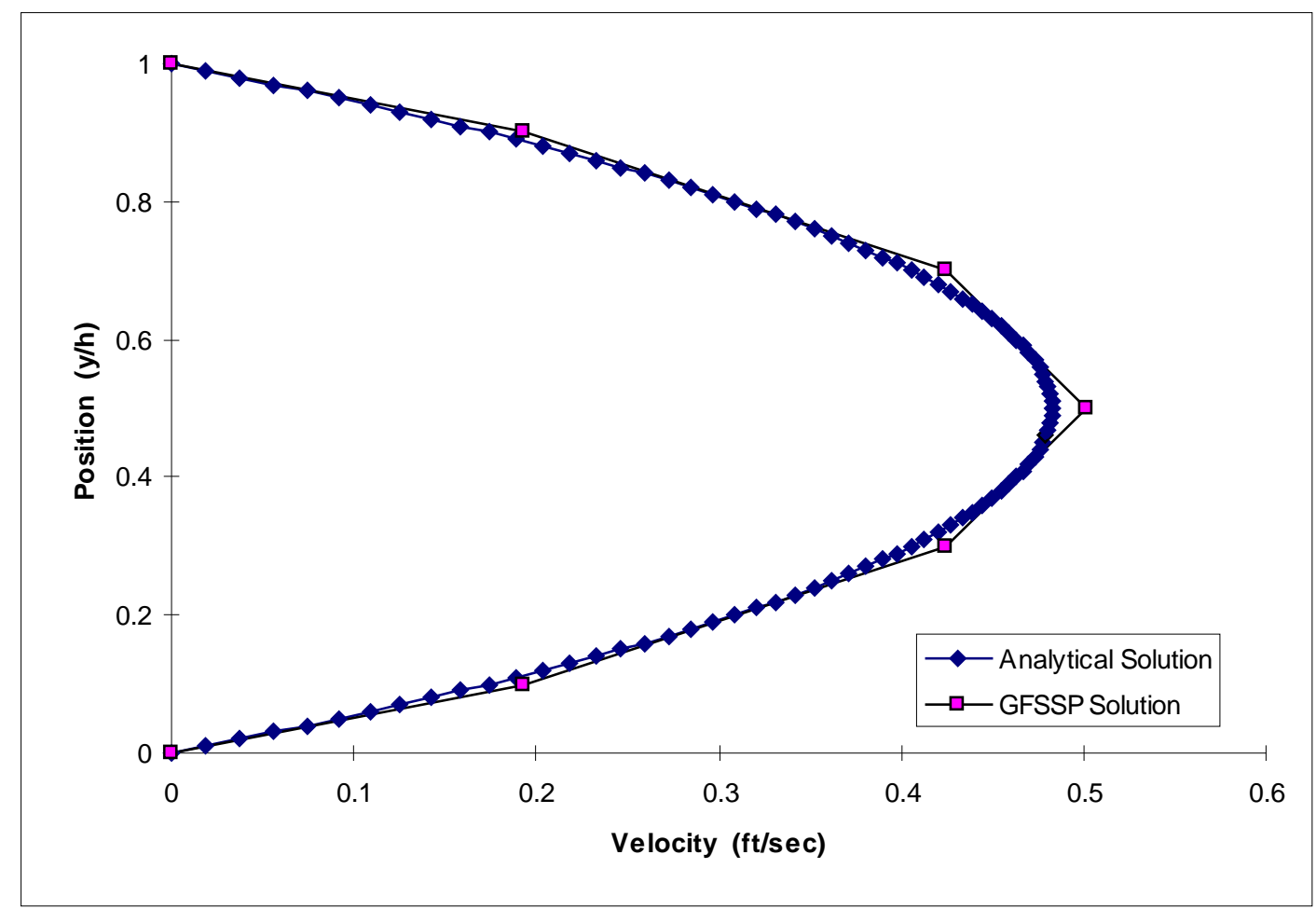

Figure 5: Poiseulle Flow Velocity Distribution

\section{COUETTE FLOW}

Consider the flow between two fixed flat plates shown in Figure 6. The flow is shear driven and assumed to be fully developed. The length of the plates is 1000 inches, they are 1 inch apart, the fluid density is $12 \mathrm{lb}_{\mathrm{m}} / \mathrm{ft}^{3}$, the viscosity is $1 \mathrm{lb}_{\mathrm{m}} /(\mathrm{ft} \cdot \mathrm{sec})$. The pressures at the upstream and downstream ends are both set at $10 \mathrm{psi}$. The bottom plate is fixed, and the top plate is moving with a velocity of $100 \mathrm{ft} / \mathrm{sec}$. Figure 7 shows an approximate velocity profile for this situation.

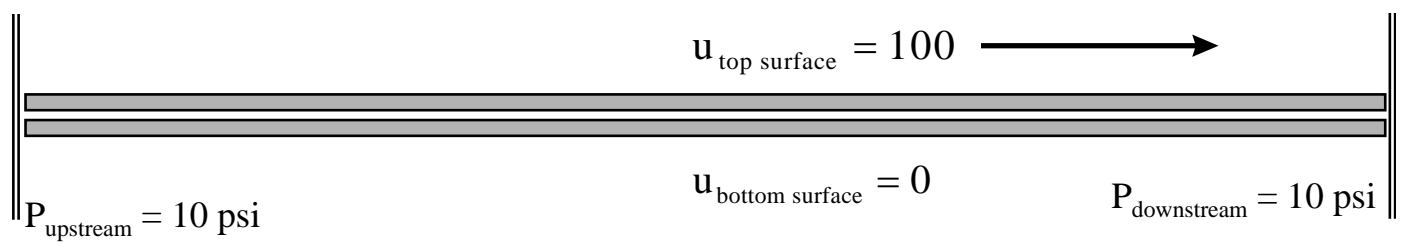


Figure 6: Couette Flow Physical Situation

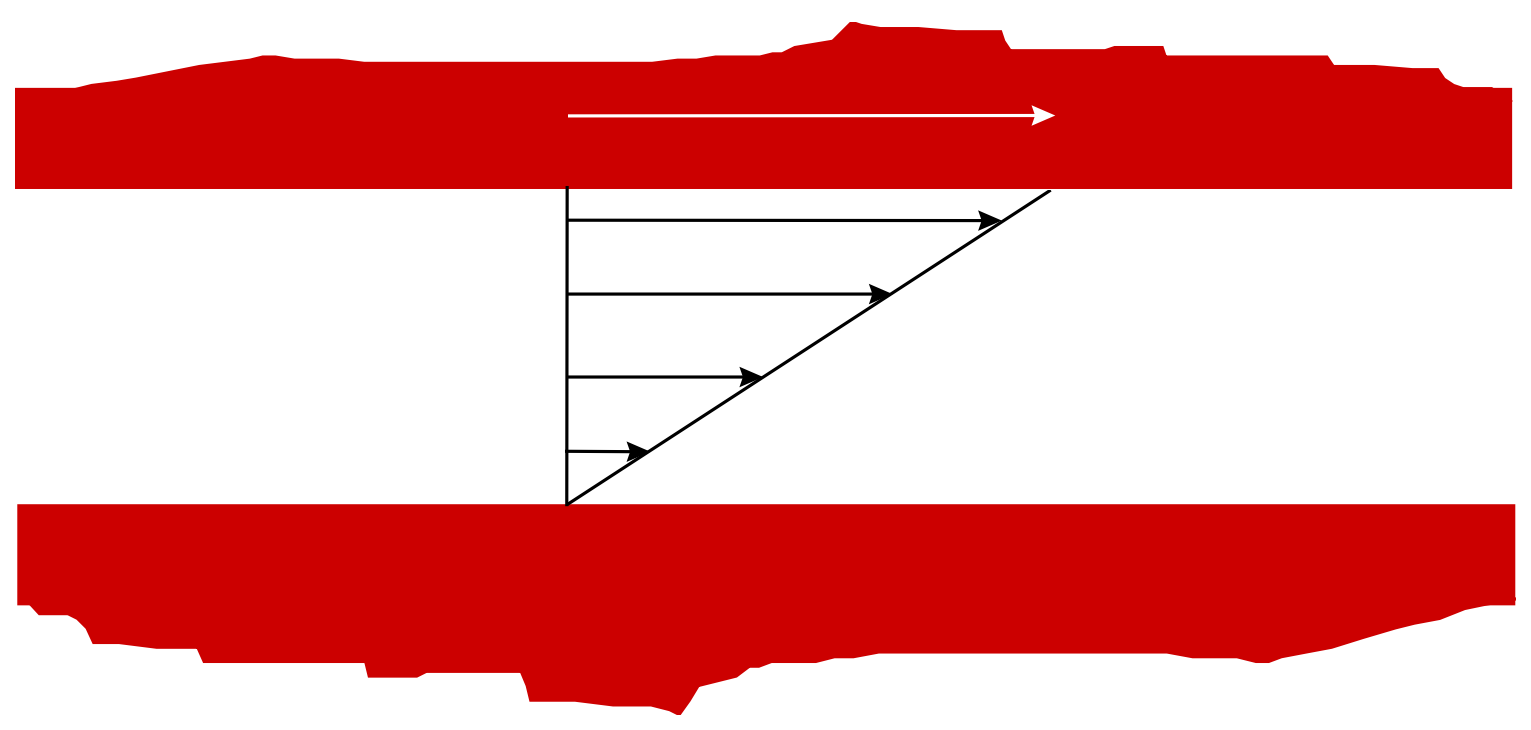

Figure 7: Couette Flow Velocity Profile

The analytical solution for Couette flow situation [4] shown in Figure 6 can be expressed as

$$
\mathrm{u}=100 \mathrm{y} \text {. }
$$

\section{GFSSP Model of Couette Flow}

A simple 3 node, 10 branch model (two sets of 5 parallel branches) was constructed to model the physical situation described above. The model is shown schematically in Figure 8 below.

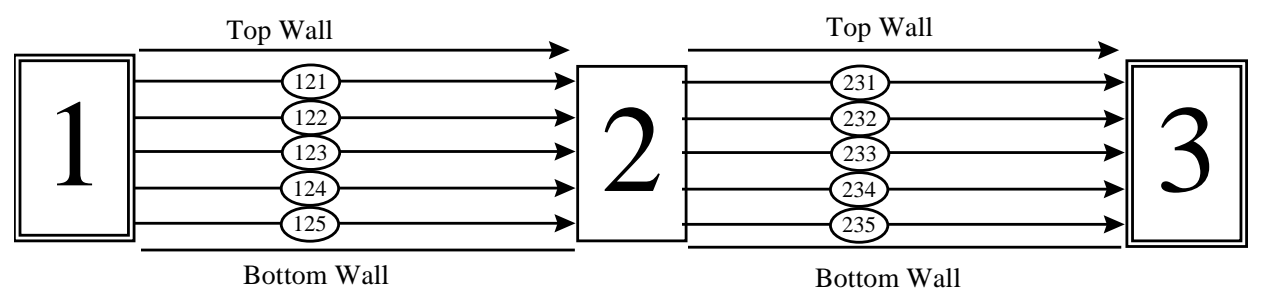

Figure 8: GFSSP Couette Flow Model

Each of the 10 branches $(121-125,231-235)$ is 500 inches in length. It is assumed that the shear area for each branch-branch \& branch-wall was 500.0 in $^{2}$ and that the shear distance was 0.2 inches between adjacent branches and 0.1 inch between walls and their adjacent branches. The pressures at nodes 1 and 3 are both $10.0 \mathrm{psi}$. A constant density option was used and the corresponding density and viscosity used were $12.0 \mathrm{lb}_{\mathrm{m}} / \mathrm{ft}^{3}$ and $1.0 \mathrm{lb} / \mathrm{m} /(\mathrm{ft} \cdot \mathrm{sec}$ ). Resistance option -02 was used in the initial flow field calculation (for the first Newton-Raphson iteration, after 
which the shear will replace the friction factor calculation) for each of the branches. The bottom walls are fixed, and the top walls are moving at a velocity of $100 \mathrm{ft} / \mathrm{sec}$.

Figure 9 below shows a comparison between the velocity profiles for the analytical solution and the GFSSP 3 node, 10 branch (5 parallel branch) model. As can be seen in Figure 9, the results of the GFSSP model compare nearly identically with the analytical solution.

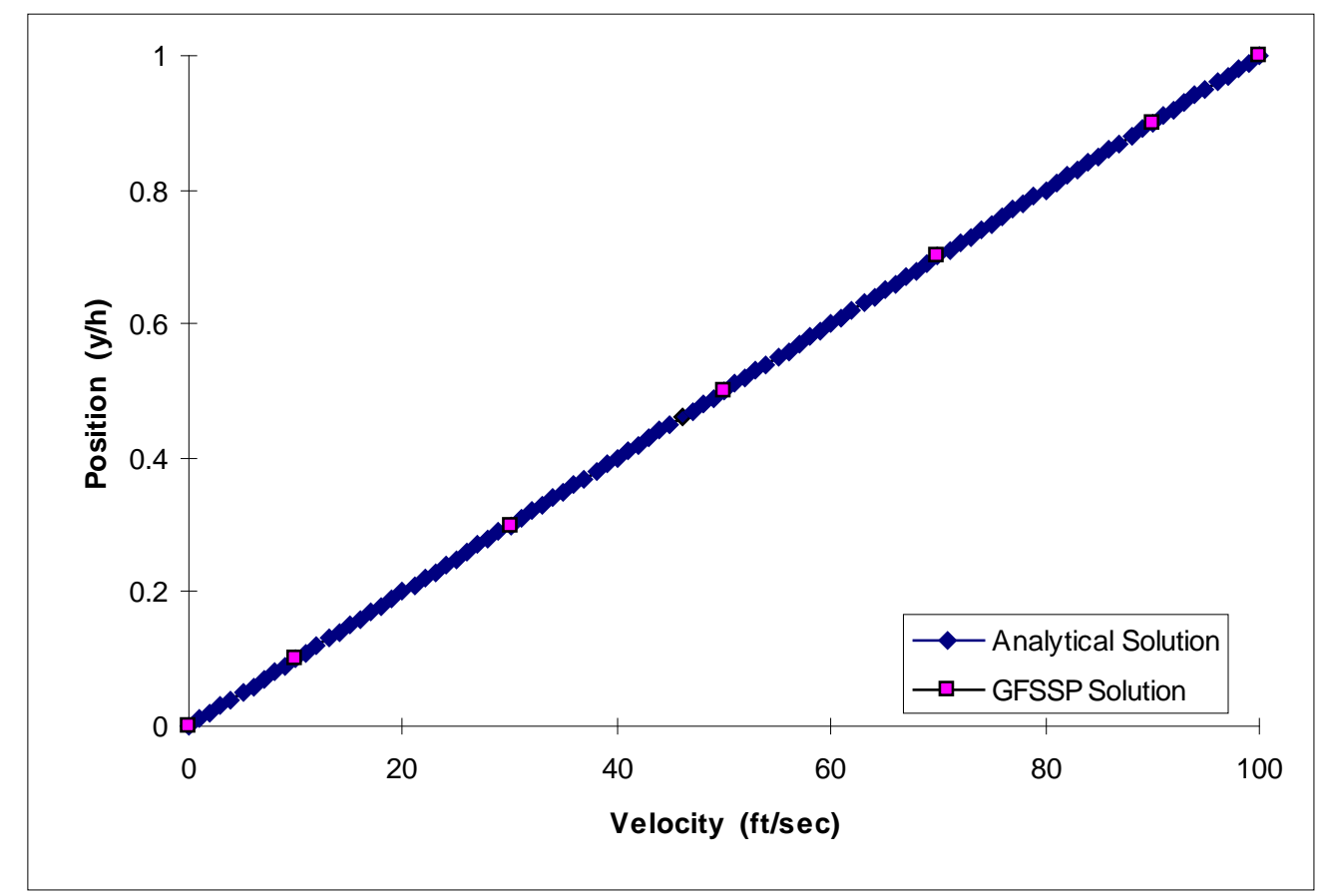

Figure 9: Couette Flow Velocity Distribution

\section{$\underline{\text { Transverse Momentum Term in Momentum Equation }}$}

The second term on the left hand side of equation (1) corresponds to the transverse momentum exchange. The discretized form of this term is expressed in equation 10.

$$
\frac{\partial \rho \mathrm{vu}}{\partial \mathrm{y}} \mathrm{V} \approx \frac{(\rho \mathrm{v} \Delta \mathrm{u})}{(\Delta \mathrm{y})}(\Delta \mathrm{x})(\Delta \mathrm{y})(\Delta \mathrm{z})=(\Delta \mathrm{x})(\Delta \mathrm{z})(\rho \mathrm{v} \Delta \mathrm{u})=\dot{\mathrm{m}}_{\text {trans }} \Delta \mathrm{u}
$$

Figure 10, below, represents a set of nodes and branches for which transverse momentum exchange will take place. Let the branch 12 represent the current branch which will receive transverse momentum from the surrounding branches. Branch S12 represents an adjacent parallel branch, while branches S1 and S2 represent the adjacent normal branches. 


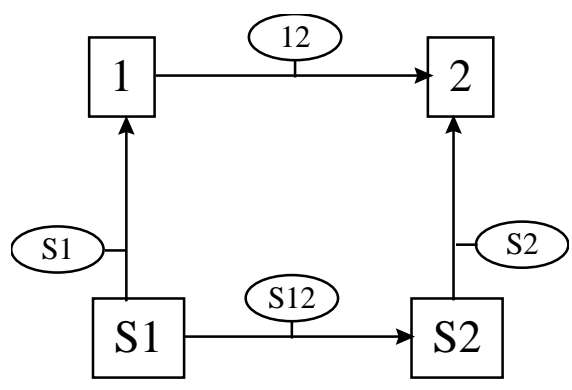

Figure 10: Grid for Transverse Momentum Exchange

Now, we will examine the formulation for calculating the transverse momentum term for branch 12. First, calculate the average mass flow rate for the adjacent normal branches:

$$
\dot{\mathrm{m}}_{\mathrm{s}}=\frac{1}{2}\left(\dot{\mathrm{m}}_{\mathrm{s} 1}+\dot{\mathrm{m}}_{\mathrm{s} 2}\right)
$$

Now, examining Figure 10 we see that a positive transverse mass flow rate is defined as flow into the nodes corresponding to the branch in question. Based on this definition of transverse mass flow rate, let's calculate the transverse momentum term:

$$
\left.\frac{\partial \rho \mathrm{vu}}{\partial \mathrm{y}} \mathrm{v}\right|_{12} \approx\left(\dot{\mathrm{m}}_{\mathrm{s}} \mathrm{u}_{12}-\dot{\mathrm{m}}_{\mathrm{s}} \mathrm{u}_{\mathrm{s} 12}\right)=\dot{\mathrm{m}}_{\mathrm{s}}\left(\mathrm{u}_{12}-\mathrm{u}_{\mathrm{s} 12}\right)
$$

The parallel branch (S12) will contribute to the transverse momentum term to branch 12 when $\dot{\mathrm{m}}_{12} \geq 0$ (since a positive transverse flow rate begins at S12 and ends at 12), and should have a negligible contribution when $\dot{\mathrm{m}}_{12}<0$ (i.e. transverse flow rate begins at 12 and ends at S12). Equation (13), below, is the upwinding representation of the transverse momentum term for branch 12.

$$
\left.\frac{\partial \rho \mathrm{vu}}{\partial \mathrm{y}} \mathrm{V}\right|_{12} \approx \mathrm{u}_{12}\left(\max \left\|-\dot{\mathrm{m}}_{\mathrm{s}}, 0\right\|\right)-\mathrm{u}_{\mathrm{s} 12}\left(\max \left\|0, \dot{\mathrm{m}}_{\mathrm{s}}\right\|\right)
$$

Equation (13) can be generalized for m-parallel branches around branch i, each with an angle $\square_{\text {j }}$ with respect to branch $i$, and $n_{i j}$ corresponding transverse connecting branches, each transverse branch with an angle of $\square_{\text {jk }}$ with respect to branch i. Equation (14) represents this generalized version of equation (13).

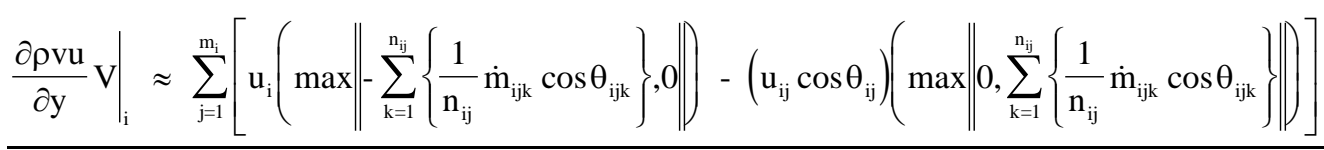


Equation (14) represents the transverse momentum formulation where $i$ is the current branch for which transverse momentum is being calculated, $\mathrm{m}_{\mathrm{i}}$ is the number of parallel branches which will be used to calculate transverse momentum, and $n_{i j}$ is the number of connecting transverse branches between the current branch (i), and the $\mathrm{j}^{\text {th }}$ parallel branch.

The implementation of Equation (14) in GFSSP has been verified by comparing its solution with benchmark solution of Burggraf for driven cavity [5].

\section{Driven Cavity Flow}

In a square cavity the flow is induced by shear interaction at the top wall as shown in Figure 11. The length of each wall is 12 inches. The density of the fluid is assumed constant at $1.00 \mathrm{lb}_{\mathrm{m}} / \mathrm{ft}^{3}$, and the viscosity of the fluid is assumed to be $1.00 \mathrm{lb}_{\mathrm{m}} /(\mathrm{ft} \mathrm{sec})$. The bottom and side walls are fixed. The top wall is moving to the right at constant speed of $100 \mathrm{ft} / \mathrm{sec}$. The corresponding Reynolds number for this situation is $\mathrm{Re}=100$.

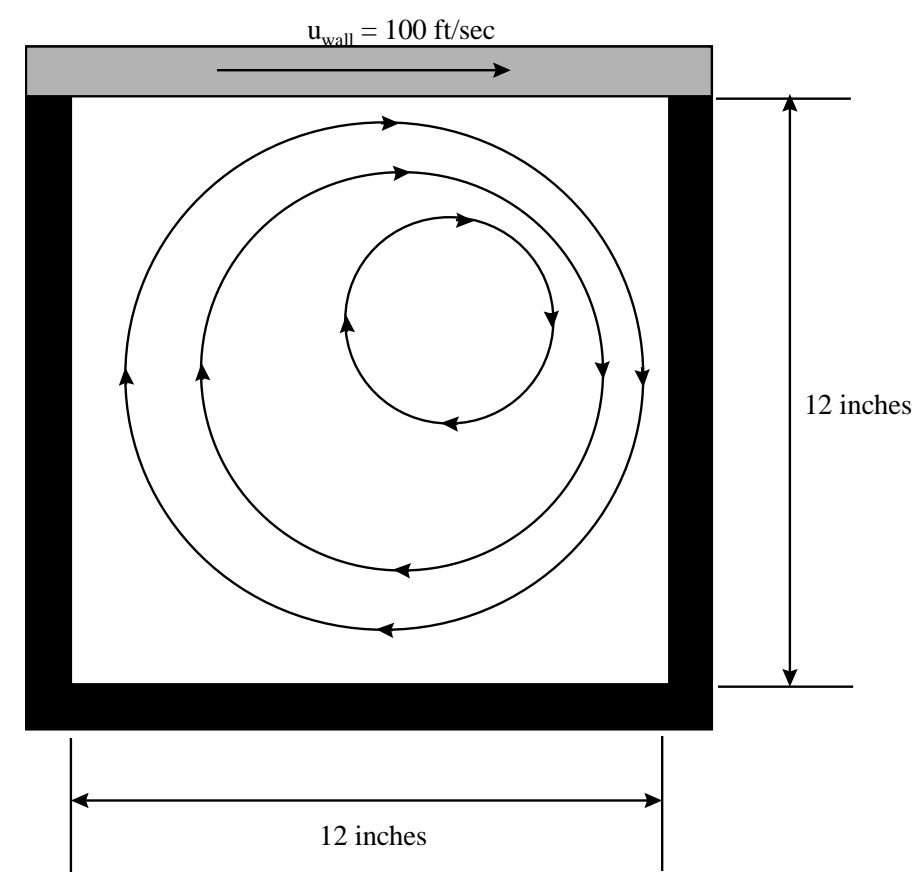

Figure 11: Flow in a Shear Driven Square Cavity

\section{GFSSP Model of Driven Cavity}

The GFSSP model of the driven cavity consists of 49 nodes (48 of which are internal) and 84 branches. For numerical stability, one node (node 1) was assigned to be a boundary node with an arbitrary pressure of 100 psi. A unit depth (1 inch) was assumed for the required areas. The model is shown schematically in Figure 12. 
It is assumed that the shear area for each branch is $2.0 \mathrm{in}^{2}$. The shear distance between adjacent branches is 1.71429 inches and the shear distance between walls and their adjacent branches is 0.85714 inches. For transverse momentum, only adjacent parallel branches were considered, and only connecting branches not associated with the boundary node were used. A constant density option was used and the corresponding density and viscosity used were $1.0 \mathrm{lb}_{\mathrm{m}} / \mathrm{ft}^{3}$ and 1.0 $\mathrm{lb}_{\mathrm{m}} /(\mathrm{ft} \mathrm{sec})$, respectively. Resistance option -02 was used in the initial flow field calculation (for the first Newton-Raphson iteration, after which the shear will replace the friction factor calculation) for all of the branches. The bottom and side walls are fixed. The top walls are moving to the right at $100 \mathrm{ft} / \mathrm{sec}$. All parallel angles are $0^{\circ}$, and all transverse angles are $90^{\circ}$.

Figure 13 shows a comparison between the benchmark numerical solution and GFSSP 7x7 node model velocity profiles along a vertical plane at the horizontal midpoint. As can be seen in Figure13, the results of this crude GFSSP model compare very favorably with the benchmark numerical solution of Burggraf [6].

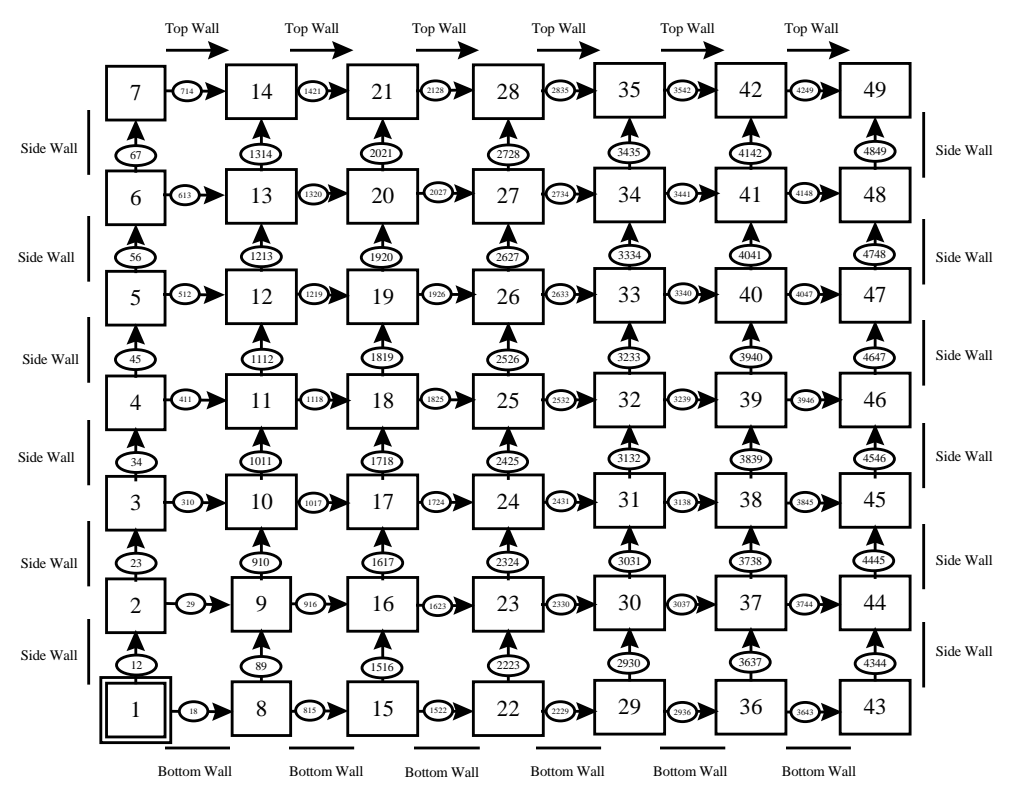

Figure 12: GFSSP Network Model of Driven Square Cavity 


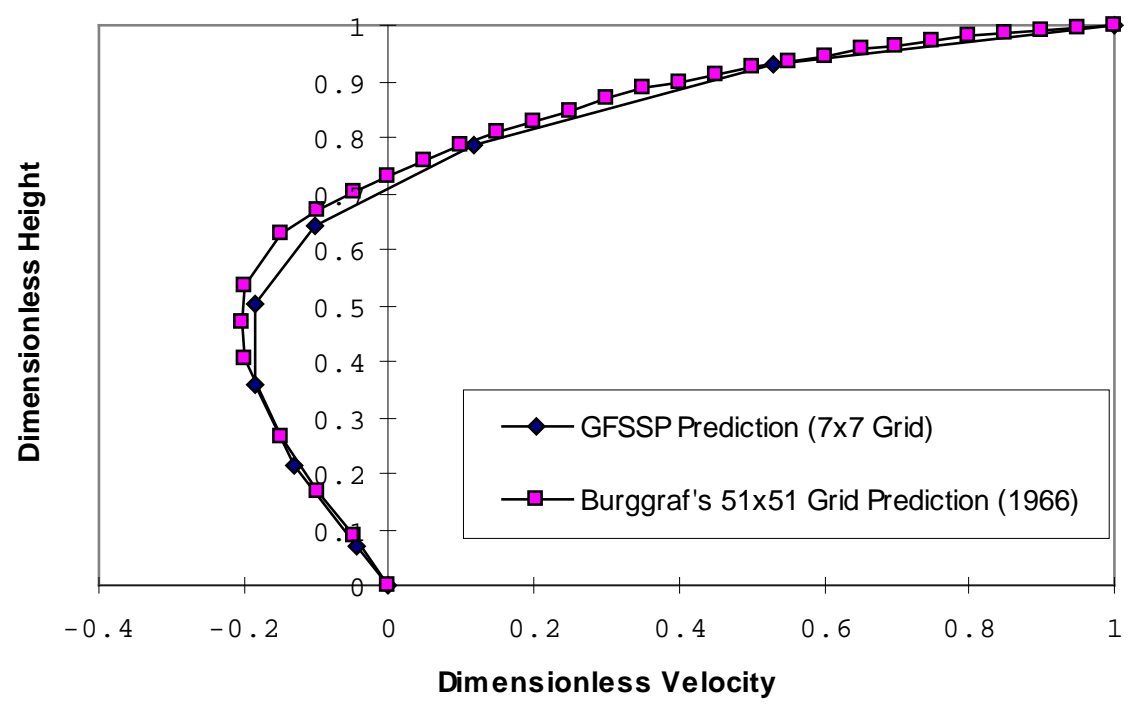

Figure 13: Shear Driven Square Cavity Centerline Velocity Distribution

The predicted velocity field and pressure contours are shown in Figure 14. The recirculating flow pattern and stagnation of flow near the top right corner are clearly shown in the figure. The predicted stream traces from calculated velocity field is shown in Figure 15.

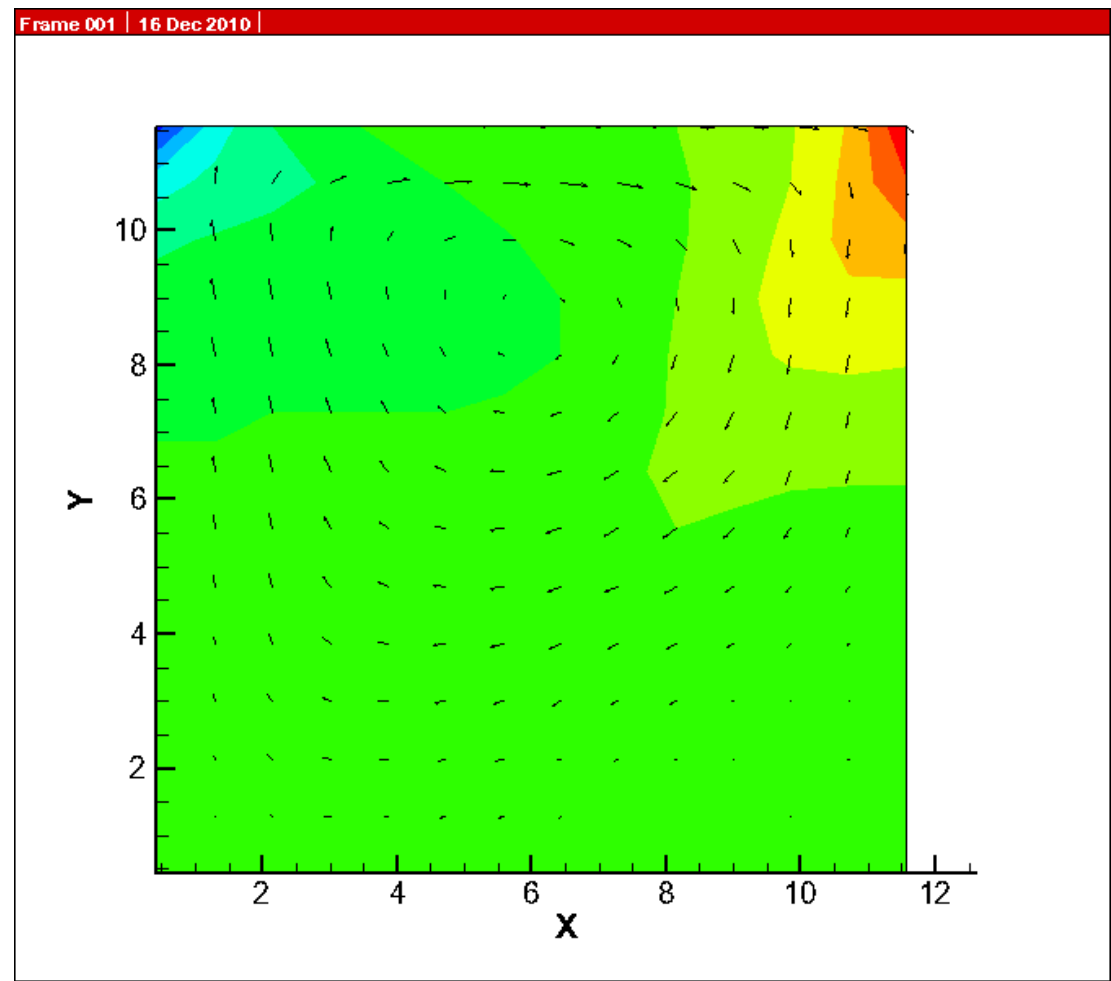

Figure 14. Predicted Velocity Field and Pressure Contours 


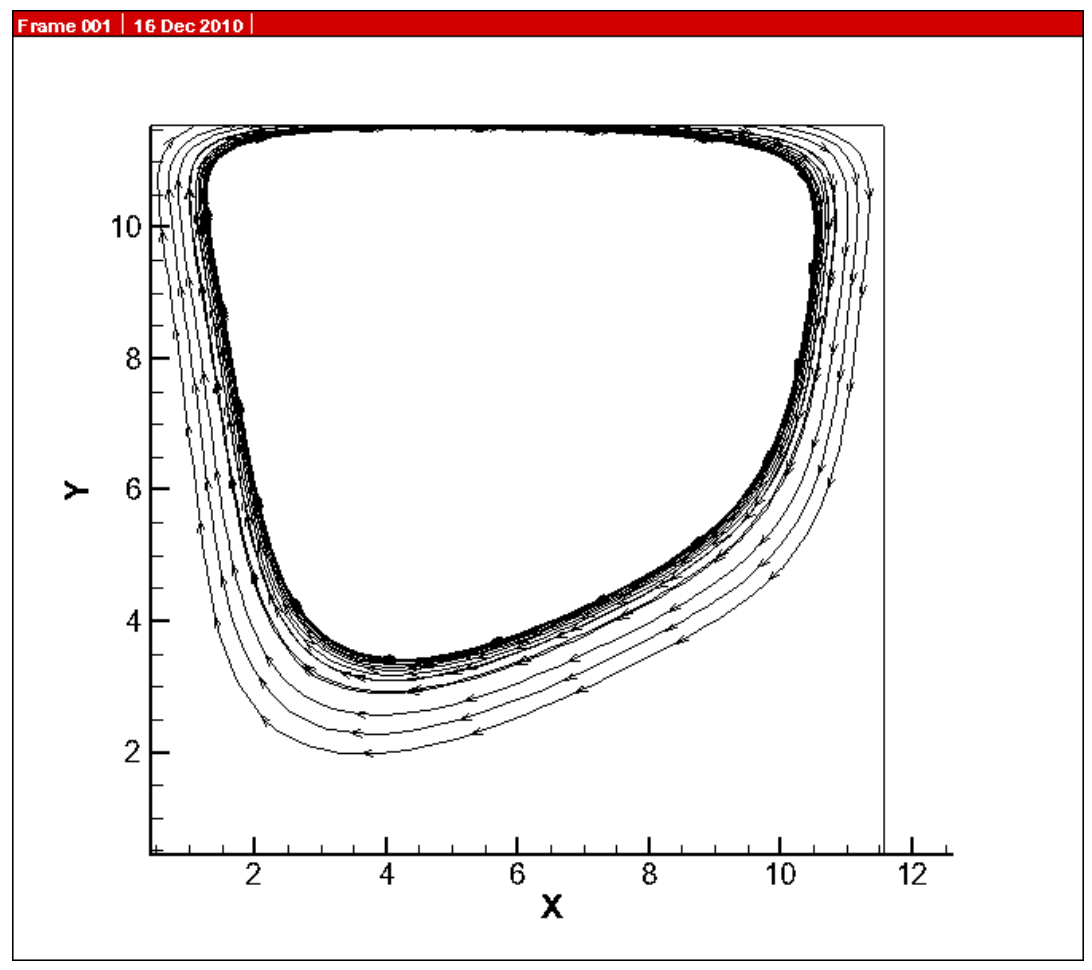

Figure 15. Predicted Stream Traces in the Driven Cavity

\section{CONCLUSIONS}

The numerical algorithm of GFSSP has been extended to calculate multi-dimensional flow. GFSSP's unstructured nodal network provides the ability to account for transport of scalar variables in n-dimensional space. Therefore, the formulation of the conservation equations for mass, energy and fluid species was not modified. However, the momentum equations are onedimensional. This paper has described the extension of the momentum equations to calculate the shear force due to velocity gradient and transport of momentum due to transverse velocity. The implementation of these terms has been validated by comparing the numerical solution with three classical benchmark problems: Poiseulle Flow, Couette Flow and flow in a driven cavity. Excellent comparison has been obtained in all three cases. Future work will include turbulent flow and heat transfer.

\section{REFERENCES}

1. Generalized fluid system simulation program, United States Patent 6748349 by Majumdar, A. K., Bailey, J.W., Schallhorn, P.A. and Steadman, T.E. June, 2004 
2. Patankar, S. V., Numerical Heat Transfer and Fluid Flow, Hemisphere Publishing Corporation, Washington, DC, 1980.

3. Majumdar, A. K., "A Second Law Based Unstructured Finite Volume Procedure for Generalized Flow Simulation,” AIAA Paper 99-0934, August 1999.

4. Schlichting, H., Boundary Layer Theory, Sixth Edition, McGraw Hill Book Company, 1968.

5. Burggraf, O.R.: "Analytical and Numerical Studies of the Structure of Steady Separated Flows”, Journal of Fluid Mechanics, Vol. 24, part 1, pp. 113-151, 1966.

\section{NOMENCLATURE}

Symbol

\section{Description}
A Area $\left(\mathrm{in}^{2}\right)$
c Constant of Integration
g Gravitational Acceleration $\left(32.174 \mathrm{ft} / \mathrm{sec}^{2}\right)$
m $\quad$ Mass Flow Rate $\left(\mathrm{lb}_{\mathrm{m}} / \mathrm{sec}\right)$
m, n Number of Branches
p Pressure (psi)
t Time (sec)
$\mathrm{u} \quad$ Fluid Velocity in the $\mathrm{x}$-Direction or in the Direction of Interest (ft/sec)
v Fluid Velocity in the y-Direction or Normal to the Direction of Interest (ft/sec)
V Volume $\left(\right.$ in $\left.^{3}\right)$
w Fluid Velocity in the z-Direction or Normal to the Direction of Interest (ft/sec)

$\mathrm{x}, \mathrm{y}, \mathrm{z}$ The Cartesian Coordinates 


\section{Greek Symbols}

$\mu \quad$ Fluid Viscosity $\left(\mathrm{lb}_{\mathrm{m}} /\left\{\mathrm{ft}^{\prime} \mathrm{sec}\right\}\right)$

$\rho \quad$ Mass Density $\left(\mathrm{lb}_{\mathrm{m}} / \mathrm{ft}^{3}\right)$

$\theta \quad$ Angle

\section{Subscripts}

$\mathrm{i}, \mathrm{j}, \mathrm{k} \quad$ Summation Indices 